POS PROCEEDINGS

\title{
The Physics of Massive Neutrinos
}

\section{M.C. Gonzalez-Garcia*}

Institució Catalana de Recerca i Estudis Avançats (ICREA) \& Departament d'Estructura $i$ Constituents de la Matèria, Universitat de Barcelona, Diagonal 647, E-08028, Spain and

Y.I.T.P., SUNY at Stony Brook, Stony Brook, NY 11794-3840, USA,

E-mail: conchadinsti.physics.sunysb.edu

In this talk I review the present status of neutrino masses and mixing and some of their implications for particle physics phenomenology.

10th International Workshop on Neutrino Factories, Super beams and Beta beams June 30 - July 52008

Valencia, Spain

${ }^{*}$ Speaker. 


\section{Introduction: The New Minimal Standard Model}

The $\mathrm{SM}$ is a gauge theory based on the gauge symmetry $S U(3)_{\mathrm{C}} \times S U(2)_{\mathrm{L}} \times U(1)_{\mathrm{Y}}$ spontaneously broken to $S U(3)_{\mathrm{C}} \times U(1)_{\mathrm{EM}}$ by the the vacuum expectation value of a Higgs doublet field $\phi$. The SM contains three fermion generations which reside in chiral representations of the gauge group. Right-handed fields are included for charged fermions as they are needed to build the electromagnetic and strong currents. No right-handed neutrino is included in the model since neutrinos are neutral.

In the SM, fermion masses arise from the Yukawa interactions which couple the right-handed fermion singlets to the left-handed fermion doublets and the Higgs doublet. After spontaneous electroweak symmetry breaking these interactions lead to charged fermion masses but leave the neutrinos massless. No Yukawa interaction can be written that would give a tree level mass to the neutrino because no right-handed neutrino field exists in the model.

Furthermore, within the SM $G_{\mathrm{SM}}^{\text {global }}=U(1)_{B} \times U(1)_{e} \times U(1)_{\mu} \times U(1)_{\tau}$ is an accidental global symmetry. Here $U(1)_{B}$ is the baryon number symmetry, and $U(1)_{e, \mu, \tau}$ are the three lepton flavor symmetries. Any neutrino mass term which could be built with the particle content of the SM would violate the $U(1)_{L}$ subgroup of $G_{\mathrm{SM}}^{\text {global }}$ and therefore cannot be induced by loop corrections. Also, it cannot be induced by non-perturbative corrections because the $U(1)_{B-L}$ subgroup of $G_{\mathrm{SM}}^{\text {global }}$ is non-anomalous.

It follows then that the SM predicts that neutrinos are strictly massless. Consequently, there is neither mixing nor $\mathrm{CP}$ violation in the leptonic sector.

We now know that this picture cannot be correct. Over several years we have accumulated important experimental evidence that neutrinos are massive particles and there is mixing in the leptonic sector. In particular we have learned that:

- Solar $v_{e}^{\prime} s$ convert to $v_{\mu}$ or $v_{\tau}$ with confidence level (CL) of more than $7 \sigma$ [1].

- KamLAND find that reactor $\bar{v}_{e}$ disappear over distances of about $180 \mathrm{~km}$ and they observe a distortion of their energy spectrum. Altogether their evidence has more than $3 \sigma$ CL [1].

- The evidence of atmospheric (ATM) $v_{\mu}$ disappearing is now at $>15 \sigma$, most likely converting to $v_{\tau}[1]$.

- K2K observe the disappearance of accelerator $v_{\mu}$ 's at distance of $250 \mathrm{~km}$ and find a distortion of their energy spectrum with a CL of 2.5-4 $\sigma$ [2].

- MINOS observes the disappearance of accelerator $v_{\mu}$ 's at distance of $735 \mathrm{~km}$ and find a distortion of their energy spectrum with a CL of $\sim 5 \sigma$ [2].

- LSND found evidence for $\overline{v_{\mu}} \rightarrow \overline{v_{e}}$. This evidence has not been confirm by any other experiment so far and it is being tested by MiniBooNE.

These results imply that neutrinos are massive and the Standard Model has to be extended at least to include neutrino masses. This minimal extension is what I call The New Minimal Standard Model.

In the New Minimal Standard Model flavour is mixed in the CC interactions of the leptons, and a leptonic mixing matrix appears analogous to the CKM matrix for the quarks. However the discussion of leptonic mixing is complicated by two factors. First the number massive neutrinos $(n)$ is unknown, since there are no constraints on the number of right-handed, SM-singlet, neutrinos. Second, since neutrinos carry neither color nor electromagnetic charge, they could be Majorana 
fermions. As a consequence the number of new parameters in the model depends on the number of massive neutrino states and on whether they are Dirac or Majorana particles.

In general, if we denote the neutrino mass eigenstates by $v_{i}, i=1,2, \ldots, n$, and the charged lepton mass eigenstates by $l_{i}=(e, \mu, \tau)$, in the mass basis, leptonic $\mathrm{CC}$ interactions are given by

$$
-\mathscr{L}_{\mathrm{CC}}=\frac{g}{\sqrt{2}} \overline{l_{i L}} \gamma^{\mu} U_{i j} v_{j} W_{\mu}^{+}+\text {h.c.. }
$$

Here $U$ is a $3 \times n$ matrix $U_{i j}=P_{\ell, i i} V_{i k}^{\ell^{\dagger}} V_{k j}^{v}\left(P_{v, j j}\right)$ where $V^{\ell}(3 \times 3)$ and $V^{v}(n \times n)$ are the diagonalizing matrix of the charged leptons and neutrino mass matrix respectively $V^{\ell^{\dagger}} M_{\ell} M_{\ell}^{\dagger} V^{\ell}=$ $\operatorname{diag}\left(m_{e}^{2}, m_{\mu}^{2}, m_{\tau}^{2}\right)$ and $V^{v^{\dagger}} M_{v}^{\dagger} M_{v} V^{v}=\operatorname{diag}\left(m_{1}^{2}, m_{2}^{2}, m_{3}^{2}, \ldots, m_{n}^{2}\right)$.

$P_{\ell}$ is a diagonal $3 \times 3$ phase matrix, that is conventionally used to reduce by three the number of phases in $U . P_{V}$ is a diagonal matrix with additional arbitrary phases (chosen to reduce the number of phases in $U$ ) only for Dirac states. For Majorana neutrinos, this matrix is simply a unit matrix, the reason being that if one rotates a Majorana neutrino by a phase, this phase will appear in its mass term which will no longer be real. Thus, the number of phases that can be absorbed by redefining the mass eigenstates depends on whether the neutrinos are Dirac or Majorana particles. In particular, if there are only three Majorana (Dirac) neutrinos, $U$ is a $3 \times 3$ matrix analogous to the CKM matrix for the quarks but due to the Majorana (Dirac) nature of the neutrinos it depends on six (four) independent parameters: three mixing angles and three (one) phases.

A consequence of the presence of the leptonic mixing is the possibility of flavour oscillations of the neutrinos. Neutrino oscillations appear because of the misalignment between the interaction neutrino eigenstates and the propagation eigenstates ( which for propagation in vacuum are the mass eigenstates). Thus a neutrino of energy $E$ produced in a $C C$ interaction with a charged lepton $l_{\alpha}$ can be detected via a CC interaction with a charged lepton $l_{\beta}$ with a probability which presents an oscillatory behaviour, with oscillation lengths given by the phase difference between the different propagation eigenstates - which in the ultrarelativistic limit is $L_{0, i j}^{\text {osc }}=\frac{4 \pi E}{\Delta m_{i j}^{2}}-$ and amplitude that is proportional to elements in the mixing matrix.

It follows that neutrino oscillations are only sensitive to mass squared differences and do not give us information on the absolute value of the masses. Also the Majorana phases do not affect oscillations because total lepton number is conserved in the process. Experimental information on absolute neutrino masses can be obtained from Tritium $\beta$ decay experiments and from its effect on the cosmic microwave background radiation and large structure formation data [3]. If neutrinos are Majorana particles their mass and also additional phases can be determined in $v$-less $\beta \beta$ decay experiments.

Besides the flavour vacuum oscillations, described above, further flavour dependent effects occur when neutrinos travel through regions of dense matter. This is so, because they can undergo forward scattering with the particles in the medium and these interactions are, in general, flavour dependent and as a consequence the oscillation pattern is modified. However the flavour transition probability still depends only on the mass squared differences and it is independent of the Majorana phases.

The neutrino experiments described above have measured some non-vanishing $P_{\alpha \beta}$ and from these measurements we have inferred all the positive evidence that we have on the non-vanishing values of neutrino masses and mixing. In the following I will derive the allowed ranges for the 

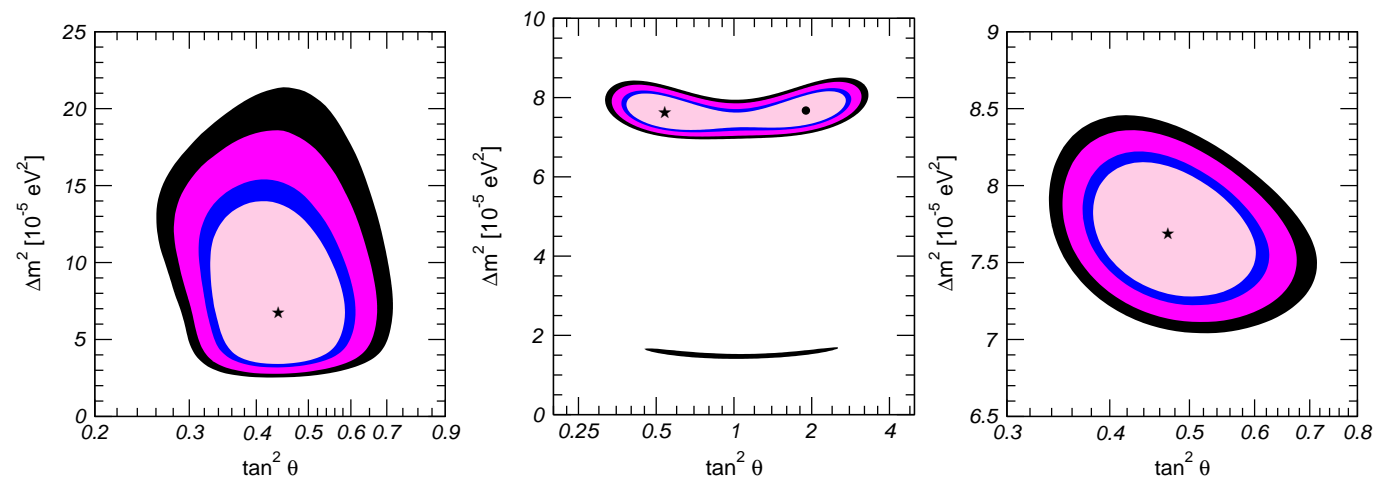

Figure 1: Allowed regions for 2-v oscillations of solar $v_{e}$ and KamLAND $\bar{v}_{e}$ and for the combination of KamLAND and solar data under the hypothesis of CPT conservation. The different contours correspond to the allowed regions at $90 \%, 95 \%, 99 \%$ and $3 \sigma \mathrm{CL}$.
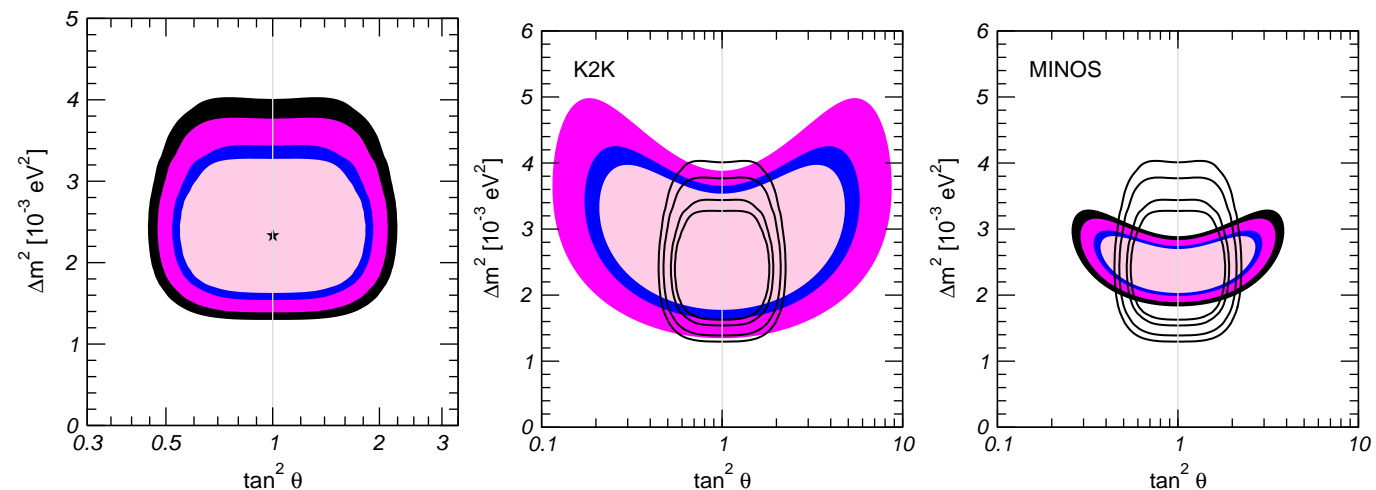

Figure 2: Allowed regions from the analysis of ATM data (left), K2K (central) and MINOS (right). The different contours correspond to at 90\%, 95\%, 99\% and $3 \sigma \mathrm{CL}$.

mass and mixing parameters when the bulk of data is consistently combined. In Fig. 2 I show the results of our latest analysis of the ATM neutrino data which includes the full data set of SuperKamiokande phases I+II.

\section{The Parameters of the NMSM: $3 v$ Analysis}

I describe here our present determination of the parameters of the model from the analysis which try to explain the evidences from solar, KamLAND, ATM and K2K experiments and assume that the LSND evidence will not be confirmed by MiniBoone.

In Fig. 1 I show the results from our latest analysis [4] of KamLAND $\bar{v}_{e}$ disappearance data, solar $v_{e}$ data and their combination under the hypothesis of CPT symmetry. The main features of these results are:

- In the analysis of solar data, only LMA is allowed at more than $3 \sigma$ and maximal mixing is rejected by the solar analysis at more than $5 \sigma$. This is so since the release of the SNO salt-data (SNOII) in Sep 2003.

- In the analysis of the KamLAND data the $3 \sigma$ region does not extend to mass values larger than $\Delta m_{21}^{2}=2 \times 10^{-4} \mathrm{eV}^{2}$ because for larger $\Delta m_{21}^{2}$ values, the predicted spectral distortions are too 
small to fit the spectral KamLAND data.

- the combined analysis allows only the LMA-I region at $3 \sigma$.

The evidence of oscillation of ATM $v_{\mu}$ has been now confirmed by two long-baseline (LBL) experiments: K2K which first observed not a deficit of $v_{\mu}$ 's at a distance of $250 \mathrm{~km}$ and in his final results also measured the distortion of their energy spectrum, and MINOS which has reported his first data in 2006 and which also observes an energy dependent deficit with a confidence level of about $\sim 5$ sigma. I show the results of our analysis of the K2K and MINOS data which graphically illustrate this agreement.

The minimum joint description of ATM, LBL, solar and reactor data requires that all the three known neutrinos take part in the oscillations. The mixing parameters are encoded in the $3 \times 3$ lepton mixing matrix which can be conveniently parametrized in the standard form

$$
U=\left(\begin{array}{ccc}
1 & 0 & 0 \\
0 & c_{23} & s_{23} \\
0 & -s_{23} & c_{23}
\end{array}\right)\left(\begin{array}{ccc}
c_{13} & 0 & s_{13} e^{i \delta} \\
0 & 1 & 0 \\
-s_{13} e^{-i \delta} & 0 & c_{13}
\end{array}\right)\left(\begin{array}{ccc}
c_{21} & s_{12} & 0 \\
-s_{12} & c_{12} & 0 \\
0 & 0 & 1
\end{array}\right)
$$

where $c_{i j} \equiv \cos \theta_{i j}$ and $s_{i j} \equiv \sin \theta_{i j}$. The angles $\theta_{i j}$ can be taken without loss of generality to lie in the first quadrant, $\theta_{i j} \in[0, \pi / 2]$.

There are two possible mass orderings, which we denote as Normal and Inverted. In the normal scheme $m_{1}<m_{2}<m_{3}$ while in the inverted one $m_{3}<m_{1}<m_{2}$.

In total the 3- $v$ oscillation analysis involves six parameters: 2 mass differences (one of which can be positive or negative), 3 mixing angles, and the CP phase. Generic 3- $v$ oscillation effects include: (i) coupled oscillations with two different wavelengths; (ii) $\mathrm{CP}$ violating effects; (iii) difference between Normal and Inverted schemes. The strength of these effects is controlled by the values of the ratio of mass differences $\Delta m_{21}^{2} /\left|\Delta m_{31}^{2}\right|$, by the mixing angle $\theta_{13}$ and by the CP phase $\delta$.

From the previous $2 v$ analysis we see that $\Delta m_{\odot}^{2}=\Delta m_{21}^{2} \ll\left|\Delta m_{31}^{2}\right| \simeq\left|\Delta m_{32}^{2}\right|=\Delta m_{\mathrm{atm}}^{2}$. As a consequence the joint 3- $v$ analysis simplifies as follows:

- for solar and KamLAND neutrinos, the oscillations with the $\Delta m_{31}^{2}$-driven oscillation length are completely averaged and the survival probability takes the form:

$$
P_{e e}^{3 v}=\sin ^{4} \theta_{13}+\cos ^{4} \theta_{13} P_{e e}^{2 v}
$$

where in the Sun $P_{e e}^{2 v}$ is obtained with the modified sun density $N_{e} \rightarrow \cos ^{2} \theta_{13} N_{e}$. So the analyses of solar data constrain three of the six parameters: $\Delta m_{21}^{2}, \theta_{12}$ and $\theta_{13}$.

- for ATM and LBL neutrinos, the $\Delta m_{21}^{2}$-driven wavelength is too long and the corresponding oscillating phase is almost negligible. As a consequence, the ATM and LBL data analysis mostly restricts $\Delta m_{31}^{2} \simeq \Delta m_{32}^{2}, \theta_{23}$ and $\theta_{13}$, the latter being the only relevant parameter common to both solar+Kamland and ATM+LBL neutrino oscillations and which may potentially allow for some mutual influence. The effect of $\theta_{13}$ is to add a $v_{\mu} \rightarrow v_{e}$ contribution to the ATM and LBL oscillations;

- at CHOOZ the $\Delta m_{21}^{2}$-driven wavelength is unobservable and the relevant oscillation wavelength is determined by $\Delta m_{31}^{2}$ and its amplitude by $\theta_{13}$.

The CP phase is basically unobservable although there is some marginal sensitivity in the present ATM neutrino analysis [4]. Normal versus Inverted orderings could be discriminated due 
to matter effects in the Earth for ATM neutrinos. However, this effect is controlled by the mixing angle $\theta_{13}$. Presently all data favour small $\theta_{13}$ with best fit point very near $\theta_{13}=0$. The dominant constraint arises from the combined analysis of CHOOZ reactor and ATM data and it is further limited by the solar and KamLAND results. Consequently, the difference between Normal and Inverted orderings is too small to be statistically meaningful in the present analysis.

Altogether the derived ranges for the six parameters at $1 \sigma(3 \sigma)$ are: The derived ranges for the six parameters at $1 \sigma(3 \sigma)$ are:

$$
\begin{aligned}
\Delta m_{21}^{2} & =7.67_{-0.21}^{+0.22}\left(\begin{array}{l}
+0.67 \\
-0.61
\end{array}\right) \times 10^{-5} \mathrm{eV}^{2}, \\
\Delta m_{31}^{2} & = \begin{cases}-2.37 \pm 0.15\left({ }_{-0.46}^{+0.43}\right) \times 10^{-3} \mathrm{eV}^{2} \quad \text { (inverted hierarchy) } \\
+2.46 \pm 0.15\left({ }_{-0.42}^{+0.47}\right) \times 10^{-3} \mathrm{eV}^{2} \quad \text { (normal hierarchy) }\end{cases} \\
\theta_{12} & =34.5 \pm 1.4\left(_{-4.0}^{+4.8}\right) \\
\theta_{23} & =42.3_{-3.3}^{+5.1}\left({ }_{-7.7}^{+11.3}\right) \\
\theta_{13} & =0.0^{+3.9}\left({ }^{+9.0}\right) \\
\delta_{\mathrm{CP}} & \in[0,360] .
\end{aligned}
$$

These results can be translated into our present knowledge of the moduli of the mixing matrix $U$ :

$$
|U|_{3 \sigma}=\left(\begin{array}{lll}
0.77 \rightarrow 0.86 & 0.50 \rightarrow 0.63 & 0.00 \rightarrow 0.22 \\
0.22 \rightarrow 0.56 & 0.44 \rightarrow 0.73 & 0.57 \rightarrow 0.80 \\
0.21 \rightarrow 0.55 & 0.40 \rightarrow 0.71 & 0.59 \rightarrow 0.82
\end{array}\right)
$$

\section{Neutrinos as Tests of Other Forms of New Physics}

Using the good description of neutrino data in terms of neutrino oscillations, it is also possible to constraint other exotic forms of new physics. In my talk I discussed also some of the results in constraining the possibility of mass varying neutrinos $[5,6,7]$ and the possibility of long-range leptonic forces [8]. In these proceedings I will summarize only the constraints which can be imposed in the violation of some fundamental symmetries. Examples of those are the violation of Lorentz Invariance (VLI) [9] induced by different asymptotic values of the velocity of the neutrinos, $c_{1} \neq c_{2}$, or the violation of the equivalence principle (VEP) [10] due to non universal coupling of the neutrinos, $\gamma_{1} \neq \gamma_{2}$ to the local gravitational potential. These forms of new physics, if nonuniversal, can also induce neutrino flavour oscillations whose main differentiating characteristic is a different energy dependence of the oscillation wavelength. For example for both VLI and VEP the oscillation wavelength decreases with energy unlike for mass oscillations. ATM neutrino events extend over several decades in energy. As a consequence they can test the presence of this effect even at the subdominant level. In Ref. [11] we performed an analysis of ATM and LBL neutrino data in terms of neutrino mass oscillations plus these new physics effects and we have concluded that the determination of mass and mixing parameters is robust under the presence of these unknown forms of new physics. Conversely, the analysis permits to impose strong constraints on the violations of these symmetries. For instance we find that at 90\% CL the possible VLI a and VEP are limited to

$$
\frac{|\Delta c|}{c} \leq 1.2 \times 10^{-24}, \quad|\phi \Delta \gamma| \leq 5.9 \times 10^{-25}
$$



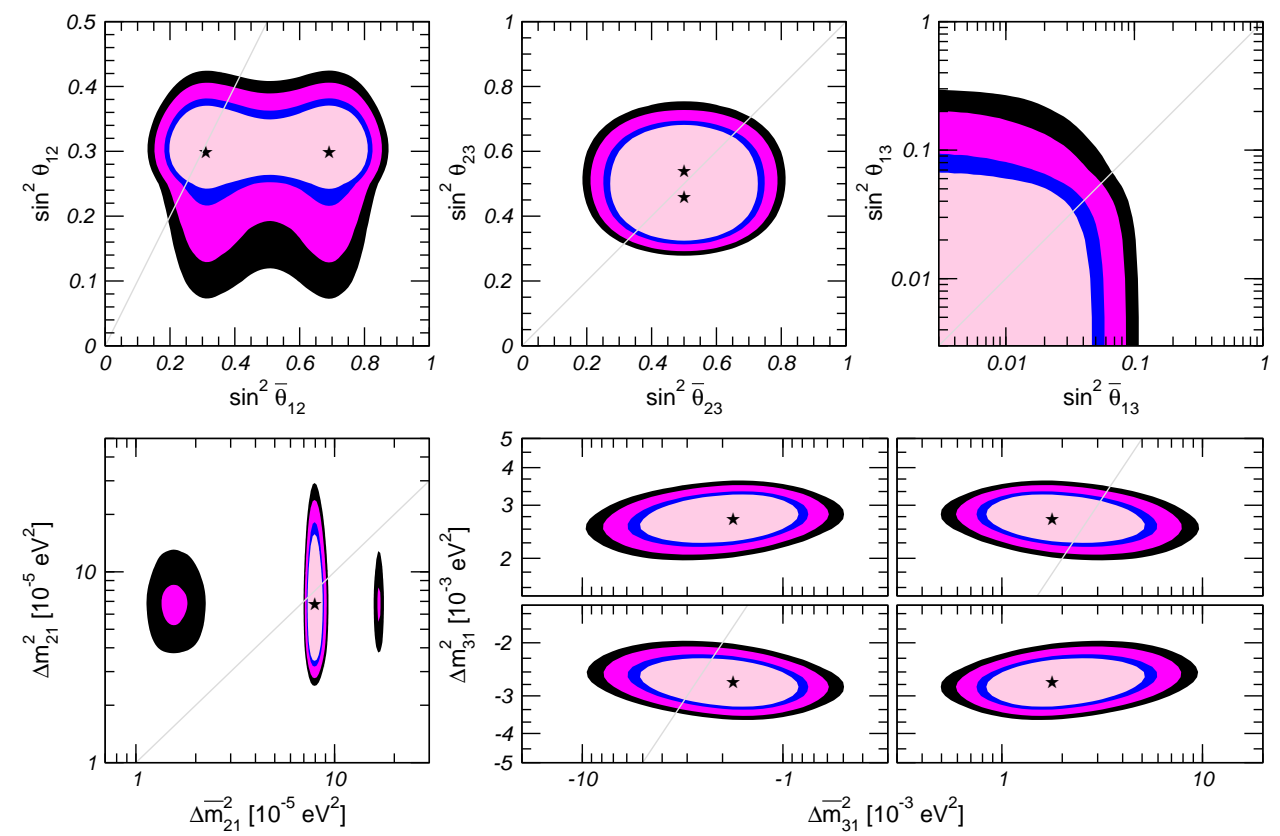

Figure 3: Allowed regions for neutrino and anti-neutrino mass splittings and mixing angles in the CPT violating scenario. Different contours correspond to the two-dimensional allowed regions at $90 \%, 95 \%$, $99 \%$ and $3 \sigma \mathrm{CL}$. The best fit point is marked with a star.

which constitute the strongest constraints on the violation of these symmetries.

As another example of the reach of the present experimental data in constraining exotic forms of new physics I comment here on alternative explanations to the LSND result which include the possibility of CPT [12] violation and imply that the masses and mixing angles of neutrinos may be different from those of antineutrinos. To test this possibility, in Ref. [13] we performed an analysis of the existing data from solar, ATM, LBL, reactor and SBL experiments in the framework of CPT violating oscillations. The outcome of the analysis is that, presently, the hypothesis of CPT violation is not supported by the data. This arises from two main facts: (i) KamLand finds that reactor $\bar{v}_{e}$ oscillate with wavelength and amplitude in good agreement with the expectations from the LMA solution of the solar $v_{e}$; (ii) both ATM neutrinos and antineutrinos have to oscillate with similar wavelengths and amplitudes to explain the ATM data. In general, as a result of these effects, the best fit to the data is very near CPT conservation as illustrated in Fig. 3.

Concerning LSND, the results show that values of $\Delta \bar{m}_{31}^{2}=\Delta \bar{m}_{\text {LSND }}^{2}$ large enough to fit the LSND result do not appear as part of the $3 \sigma \mathrm{CL}$ allowed region of this all-but-LSND analysis. It is bounded to $\Delta \bar{m}_{31}^{2}<0.01 \mathrm{eV}^{2}$ with this upper bound being determined by atmospheric neutrino data. It is clear from these results that the CPT violation scenario cannot give a good description of the LSND data and simultaneously fit all-but-LSND data.

\section{Acknowledgments}

This work is supported by National Science Foundation grant PHY-0354776 and by Spanish Grants FPA-2007-66665-C02-01, and FPA2006-28443-E. 


\section{References}

[1] T. Kajita, Solar and Atmospheric Neutrino Experiments, in proceedings of 10th International Workshop on Neutrino Factories, Super beams and Beta beams (NuFact08), June 30 - July 5 2008, Valencia, Spain PoS (NUFACT08) 006

[2] N. Saoulidou, Present and Future Long baseline Experiments, in proceedings of 10th International Workshop on Neutrino Factories, Super beams and Beta beams (NuFact08), June 30 - July 5 2008, Valencia, Spain PoS (NUFACT08) 003

[3] S. Pastor, Neutrinos in Cosmology, in proceedings of 10th International Workshop on Neutrino Factories, Super beams and Beta beams (NuFact08), June 30 - July 5 2008, Valencia, Spain POS (NUFACT08) 025

[4] M. C. Gonzalez-Garcia and M. Maltoni, Phys. Rept. 460, 1 (2008).

[5] R. Fardon, A. E. Nelson and N. Weiner, JCAP 0410, 005 (2004); D. B. Kaplan, A. E. Nelson and N. Weiner, Phys. Rev. Lett. 93, 091801 (2004).

[6] M. Cirelli, M. C. Gonzalez-Garcia and C. Pena-Garay, Nucl. Phys. B 719, 219 (2005).

[7] M.C. Gonzalez-Garcia M C, P.C. de Holanda and R. Zukanovich Funchal Phys. Rev. D 73033008 (2006).

[8] M. C. Gonzalez-Garcia, P. C. de Holanda, E. Masso and R. Zukanovich Funchal, JCAP 0701 (2007) 005 .

[9] S. Coleman and S.L. Glashow, Phys. Lett. B 405, 249 (1997); D. Colladay and V.A. Kostelecky, Phys. Rev. D55, 6760 (1997).

[10] M. Gasperini, Phys. Rev. D 38 (1988) 2635; Phys. Rev. D 39, 3606 (1989)

[11] M. C. Gonzalez-Garcia and M. Maltoni, Phys. Rev. D 70, 033010 (2004)

[12] H. Murayama, and T. Yanagida, Phys. Lett. B 520, 263 (2001); G. Barenboim et al., JHEP 0210 (2002) 001.

[13] M. C. Gonzalez-Garcia et al. Phys. Rev. D 68, 053007 (2003). 